\title{
Slow Evolution of a System of Satellites Induced by Dynamical Friction
}

\author{
Serena E. Arena and Giuseppe Bertin \\ Dipartimento di Fisica, Università degli Studi di Milano, \\ via Celoria 16, I-20133 Milano, Italy \\ E-mail: serena.arena@unimi.it, giuseppe.bertin@unimi.it
}

\begin{abstract}
The evolution induced by dynamical friction on a spherical shell of rigid satellites interacting directly with the particles sampling the host elliptical galaxy is followed by means of $N$-body simulations for a variety of shell-galaxy configurations.
\end{abstract}

Keywords. stellar dynamics, methods: n-body simulations, galaxies: kinematics and dynamics

\section{Introduction}

Elliptical galaxies often host significant systems of globular clusters. The properties of the clusters change with time because of stellar evolution, internal stellar dynamical effects, and tidal interactions with the host galaxy. In addition, the distribution and kinematics of such systems can evolve because of the dynamical friction felt by the individual globular clusters while moving through the host galaxy.

Here, by means of $N$-body simulations, we focus on the evolution induced by dynamical friction by considering a system of rigid satellites initially placed on a quasi-spherical shell. With respect to the study of friction on a single heavy object, this configuration represents an intermediate step in the direction of studying a realistic three-dimensional distribution of globular clusters, with the advantage of defining a dynamical problem in which quasi-spherical symmetry is preserved in the course of evolution and of testing to what extent dynamical friction can be thought of as a basically local process. We consider a variety of such shell configurations, characterized by different mass and phase space properties, and investigate their evolution in realistic galaxy models.

The realistic galaxy models adopted are a one-parameter family, derived from the $f^{(\nu)}$ distribution function, with a central isotropic core and a radially anisotropic envelope (Trenti \& Bertin (2005)). The satellites are rigid Plummer spheres of radius $R_{f}$, initially distributed in a spherical shell of thickness $2 R_{\text {shell }}$, positioned at a distance $r_{\text {shell }}(0)$ from the galaxy center. We have considered two configurations of the shell. The first has a density profile given by $\rho_{\text {shell }}(r)=\rho_{\text {shell }} e^{-4\left[\left(r-r_{s h e l l}(0)\right) / R_{\text {shell }}\right]^{2}}$, with satellites on circular orbits; in the second configuration, satellites move on eccentric orbits with positions and velocities extracted from the distribution function of the galaxy.

The evolution of the system is followed by means of the $N$-body collisionless (meanfield) particle-mesh code described in Trenti (2005). The galaxy is made of $2.5 \times 10^{5}$ particles and the shell of $N_{f}=20$ and 100 satellites. For further details, see Arena \& Bertin (2007) and Arena (2007).

\section{The evolution induced in the spherical shell of satellites}

Each satellite loses energy and angular momentum because of dynamical friction with the particles composing the galaxy (that represent stars and/or dark matter). As a re- 

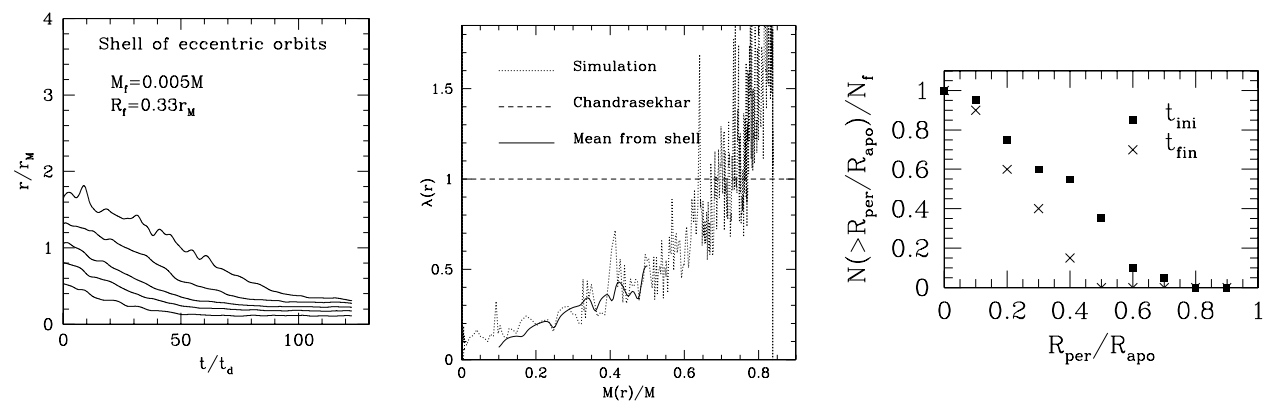

Figure 1. Left frame: evolution of the Lagrangian radii for the mass distribution of a shell made of satellites on eccentric orbits. Middle frame: ratio $\lambda$ of the Coulomb logarithm measured in the simulations to that predicted by the Chandrasekhar theory; the dotted line refers to a single satellite. Right frame: evolution of the eccentricity of the satellite orbits, see text for details. The scales $r_{M}, M$, and $t_{d}$ are the host galaxy half-mass radius, mass, and dynamical time.

sult, the shell moves slowly towards the center of the galaxy, while its thickness decreases (left frame of Fig. 1). In the final stage of evolution, the shell settles down in a quasi-equilibrium configuration without actually reaching the galaxy center. Shells made of more massive or less extended satellites remain at a larger distance from the galaxy center. The shell thickness decreases more for shells made of less extended satellites. Shells starting at different distances from the galaxy center reach the same final quasi-equilibrium configuration. The fall time observed in the simulations is longer than that predicted by the Chandrasekhar theory of dynamical friction; indeed, the measured Coulomb logarithm is smaller (middle frame of Fig. 1).

In the simulation of shells with satellites on eccentric orbits, we have studied the process of circularization. The right frame of Fig. 1 illustrates the distribution of the ratio of the pericenter to the apocenter $R_{\text {per }} / R_{\text {apo }}$ for the satellite orbits. The quantity $N\left(>R_{\text {per }} / R_{\text {apo }}\right)$ is the number of satellites with ratio $R_{p e r} / R_{a p o}$ greater than the value shown on the $\mathrm{x}$-axis. By comparing the initial distribution of points (filled squares) with that of the final configuration (crosses), we conclude that the number of satellites with eccentric orbits increases during evolution. The host galaxy responds non-adiabatically to the infalling shell with a decrease of its density concentration and of its pressure anisotropy in the radial direction (Arena et al.(2006)).

The characteristics of the entire process depend on the properties of the host galaxy, especially on its density concentration. Indeed, in low-density concentration galaxy models (e.g. polytropes; see Bertin, Liseikina \& Pegoraro (2003)) the final configuration of the shell is characterized by a larger thickness and a larger distance from the galaxy center with respect to the evolution in models with higher density concentration (e.g. the $f^{(\nu)}$ models). The study of the evolution induced by dynamical friction of a shell of satellites is a first step towards the study of the interaction with the host galaxy of a more realistic three-dimensional distribution of satellites.

\section{References}

Arena, S. E., Bertin, G., Liseikina, T., \& Pegoraro, F. 2006, A\& A, 453, 9

Arena, S.E., \& Bertin, G. 2007, A\& A, 463, 921

Arena, S. E. 2007, Ph.D. Thesis, Università degli Studi di Milano, Milano

Bertin, G., Liseikina, T., \& Pegoraro, F. 2003, A\&A, 405, 73

Trenti, M. 2005, Ph.D. Thesis, Scuola Normale Superiore, Pisa

Trenti, M., \& Bertin, G. 2005, A\&SA, 429, 161 